(Aus der experimentell-biologischen Abteilung des pathologischen Instituts der (niversität Berlin.)

\title{
Experimenteller Beitrag zur Wiederkehr des Knie- phänomens nach Pyramidenläsion bei Tabes dorsalis.
}

Von

\author{
Dr. N. OSSOKIN.
}

Fälle von zerebralen Apoplexien im Verlauf der Tabes, die schon an and für sich eine relativ nicht seltene Erscheinung sind, erlangen besonderes Interesse in physiologischer Beziehung dann, wenn die infolge des tabischen Prozesses verschwundenen Sehnenreflexe nach dem Eintritt der Hemiplegie wiederkchren. Eine solche Wiederkehr der Reflexe fand in den Fällen von Jackson, Marinesco, T'aylor, Raichlin, Goldflam, Mamlock, Westphal u. A. statt, wobei die Mehrzahl der aufgezählten Autoren'), desgleichen Sternberg in seiner großen, der Frage der Sehnenreflexe gewidmeten Monographie bei der Erklärung dieser Erscheinung von der Annahme ausgeht, daß das Fehlen der Kniereflexe in viclen Fällen, namentlich bei nicht besonders stark fortgeschrittenem Tabes-

1) Jackson, Brit. med. Journ. 189:.

Marinesco, Soc. de biol. 1893.

Taylor. Brit. med. Journ. 1894.

Raichlin. Soc. de biol. 1893. Zit. nach Cestan.

Goldflam, Berl. klin. Woch. 1891.

Mamlock, Zeitschr. f. klin. Med. Bcl. 43.

A. Westphal, Charité-Annalen. 1899-1900.

Sternberg, Dic Sehnenreflexe und ihre Bedeutung für die Pathologie des Nervensystems. 1893. 
prozeß hauptsächlich dureh die hemmenden binfliisse hedingt. ist, die rom Gehirn \%um reflelitorisehen Zerelorospinel\%ontsum dep Pyramidenbilun entlang gehent. alls welchem firmele heim

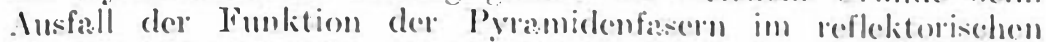

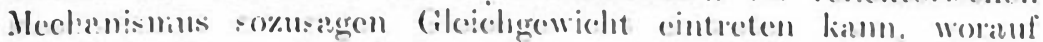
die Iniereflexe wickerlichrent.

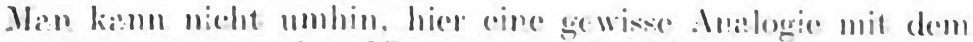

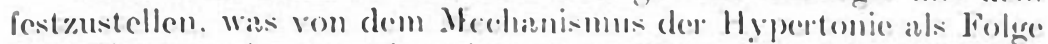

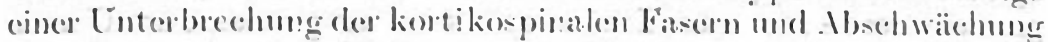

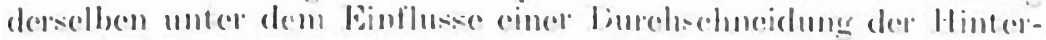

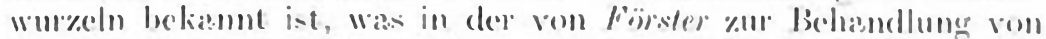

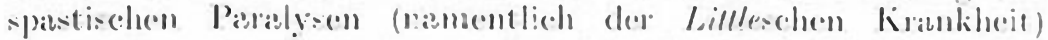

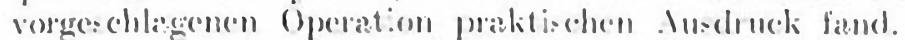

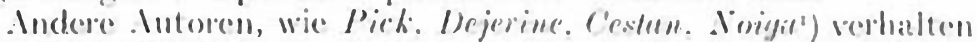

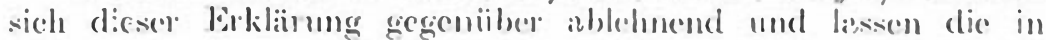

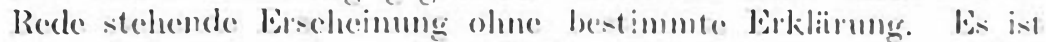

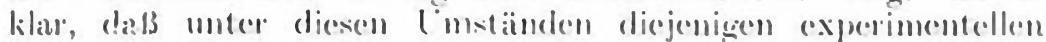
Beobarehtumgen von besonderem Interesse soin miissen, die den in Rede stehenden klinischen Fällen imbleg sind. Bekiunntlich

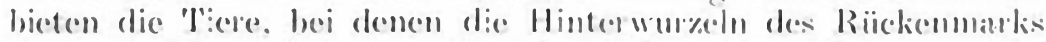

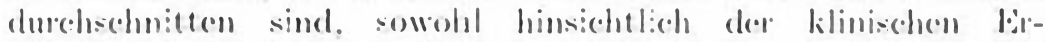

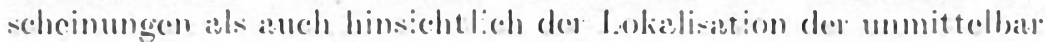

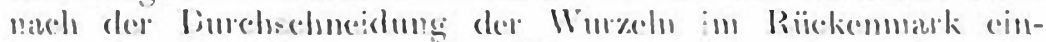

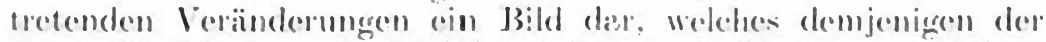

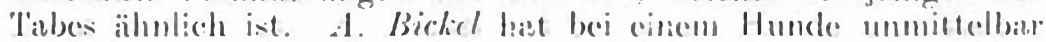

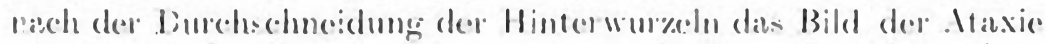

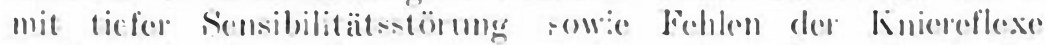

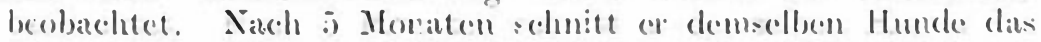
Rï̈ckenmark im oberen Brustejl durch. und nun kehten unmittelhas rach der \%weiten Operation die. Roflexe \%uriek.

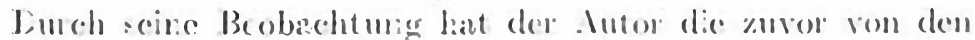

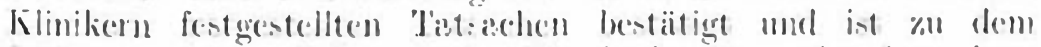

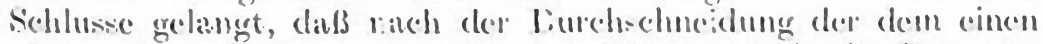

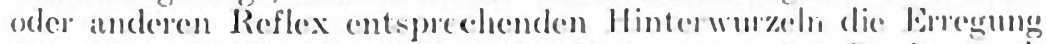
ron dem peripherischen sensiblen Neuren ant dis Rïrekenmank medh den fiasern contlang iibertragen werelon kimn, die den benachbalten segmenten angehören. Augenecheonlioh correicht diese.

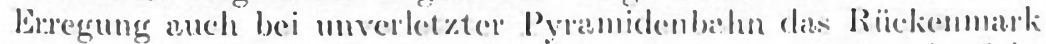

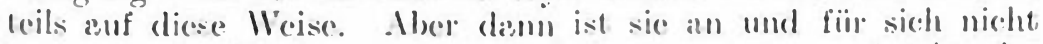
imstande, dans Rü̈ckemm:ank \%u hecinflussent. mel \%wiu infolge des hemmerelen binflusses von soiten des Gohirns.

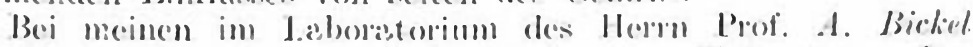

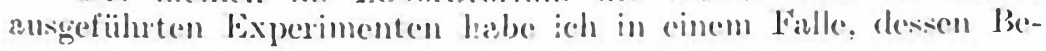

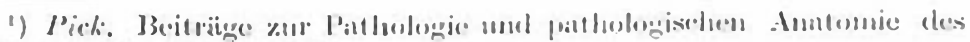

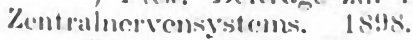

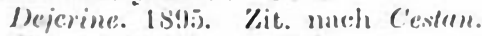

Cesterr. Isc l'rogricis micel.

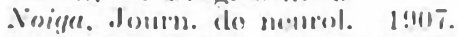

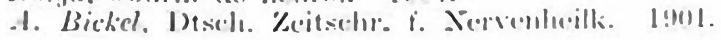


schreibung im nac:hstelenden folgt, eine nene Best:ätigung für diss erhalten, Was oben vom Mechanismus der Wiederkehr der erloschenen S'ehnenreflexe gesiegt wurde. Die Win\%elheiten dieses Experiments sind kilr\% folgende:

Am 13. VI. 1!110 wurden einem mittelgiolisen Hunde in SetherMorphium-Ninkose die 2.-5. Lumbalwurel beiderseits durchschnitten. Niuch der Operation komnte der Hund sich nicht mehr

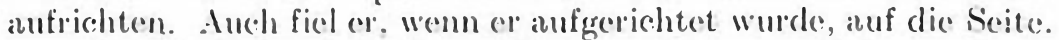

14. VI. Beim Aufstehen stiityte er sich mur auf die vorderen lixtremitäten. die hinteren befanden sich in spastischem /ustande und schleiften boi den Bewignungen des 'Tieres am Boden.

Im Bereich der hinteren batremitäten ist die Sensibilitit. erlosehen. Die Kinicueflexe fohlen gleichfalls.

2.2. VI. Die Wunde heilt regelmä̈big. Vonseiten der hinteren Extremitäten fast vollständige Paraplegic. Kniceflexe fehlen.

27. VI. Der Hund begimt sich anch anf die hinteren Extremitäten \% stuit\%en. hält sie alser stets mit der Dorsalfläche dem Boden \%ugewandt. Beim Gehen schleifen die hinteren bitremitäten nach wie vor am Boden. Knicertexe fohlen.

4. VII. Die ataktischen Störungen haben unwexentlich nachgelassen. Kniereflexe fehlen.

5. VIl. In Aether-M(s)phimm-Niukose wurde mittels (ialvamokianters nach der Methode von Rollhmemn eine P'yamidenläsion in cler Medullar oblongaltir hervergeblareht.

Zwei Stunden nach der (Operation lionnte sich der Hund nicht anfrichten: or kiat\%te bei Aufrichtungsversuchen hilflos mit den P'foten am Bod(n. Disscelbe wurde am Ibend desselben 'T'ages, fïnf Stunden nawh der Operation. konstatiert. An boiden seiten sind die Kniereflexe wiedergelielıt.

(i. VII. Der Hund \%eigt noch keine regelmäbiggen Bowegungen in den bxtremitäten. vermang aber eine ihm lejgebrachte

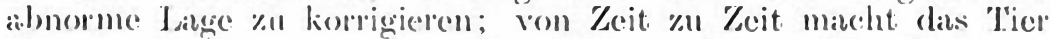
Rotationsbewegungen 1 m seine Körperachse. Die Richtung dieser Bowegungen ist jedoch von links nach rechts. Die Kniereflexe lassen sich hervolunfen, man kimn sie abel nicht als gesteigert betrakchten. In den vorderen bxtremitäten sind dic Reflexe gesteigert.

7. Vil. Der Hund beginnt $\%$ gehen, indem er sich regelmäßig auf die P’almamberfä̈le der vorderen lixtremitäten stït\%t. Die hinteren lixtremitäten befinden sich in spastischem Zustande und sind an der Lokomotion alitiv fast gar nicht betciligt. rufen.

Die Iniereflexe lassen sich an beiden bxtremitäten hervor-

11. Vll. In der Silkrilgegend stellt sich Dekubitus ein. Beim Gehen funktionieren mu die voleleren lixtremitïten, während die hinteren am Boden schleifen. Schmerygefiihl und Muskelsinn bleiben nach wie vor sestërt.

1.2. VII. Der Dokubitus entwickelt sich weiter; dor Hund verfällt. 
12. VII. Tod.

Autopsie. Die Operationsöffnungen sind im Lumbalteil der Wirbelsäule und in der Höhe der Medulla oblongata verwachsen. In der Höhle des Canalis verteb:alis fand sich Eiter nicht vor. Die Medulla oblongata wurde samt dem Rückenmark aus dem Wirbelkanal herausgeholt, in Müllerscher Flüssigkeit fixiert und daun nach der Methode von Marchi bearbaitet. Außerdem wurden einige Segmente nash der Methode ron Busch bearbeitet.

Die mikroskopische Untersuchung ergab, daß die Verletzung in der Gegend der Medulla oblongata in der Höhe der Pyramidenkreuzung den linken Vorderstrang einnahm und von hier aus in schräger Richtung zum rechten Vo:derhorn verlief.

Infolge dieser Lokalisation durchschnitt die Verletzung die Fasern der beiden Pyramiden. Anf den von den höher liegenden Segmenten der Medulla oblongata gefertigten Schnitten konnte man noch eine Verletzung der rechten Pyramide und der rechten Olive im Mittelteil derselben wahrnehmen.

Im Rückenmark waren in aufsteigender Richtung die Fasern der beiden Hinterstränge, in absteigender Richtung die Fasern der Vorderseitenstränge und der Pyramidenseitenstrangbahnen gleichmäßig auf bsiden Seiten degeneriert, wobei bemerlit werden muß, daß das beschriebene Bild in den nach $M$ archi baa!bieteten Präparaten deutlicher hervortritt als an denjenigen Präparaten, die nach Busch bearbaitet wa:en. 